\title{
Fourth-Line Therapy in Metastatic Renal Cell Carcinoma (mRCC): Results from the International mRCC Database Consortium (IMDC) ${ }^{1}$
}

Igor Stukalin $^{\mathrm{a}}$, J. Connor Wells ${ }^{\mathrm{a}, \mathrm{b}}$, Anna Fraccon ${ }^{\mathrm{c}}$, Felice Pasini $^{\mathrm{d}}$, Camillo Porta ${ }^{\mathrm{e}}$, Aly-Khan A. Lalanif ${ }^{\mathrm{f}}$, Sandy Srinivas ${ }^{\mathrm{g}}$, I. Alex Bowman ${ }^{\mathrm{h}}$, James Brugarolas ${ }^{\mathrm{i}}$, Jae-Lyun Lee ${ }^{\mathrm{j}}$, Frede Donskov ${ }^{\mathrm{k}}$, Benoit Beuselinck ${ }^{\mathrm{l}}$, Aristotelis Bamias ${ }^{\mathrm{m}}$, Brian I. Rini ${ }^{\mathrm{n}}$, Hao-Wen Sim ${ }^{\mathrm{o}}$, Neeraj Agarwal ${ }^{\mathrm{h}}$, Sun-Young Rha ${ }^{\mathrm{p}}$, Ravindran Kanesvaran ${ }^{\mathrm{q}}$, Toni K. Choueirif ${ }^{\mathrm{f}, 2}$ and Daniel Y.C. Heng ${ }^{\mathrm{a}, 2, *}$

${ }^{\mathrm{a}}$ Tom Baker Cancer Center, Calgary, AB, Canada

${ }^{\mathrm{b}}$ Queen's School of Medicine, Kingston, ON, Canada

${ }^{\mathrm{c}}$ Casa di Cura Pederzoli, Peschiera Del Garda, Italy

${ }^{\mathrm{d}}$ Ospedale Santa Maria della Misericordia, Rovigo, Italy

e IRCCS San Matteo University Hospital Foundation, Pavia, Italy

${ }^{\mathrm{f}}$ Dana-Farber Cancer Institute, Boston, MA, USA

${ }^{\mathrm{g}}$ Stanford Medical Center, Stanford, CA, USA

${ }^{\mathrm{h}}$ Huntsman Cancer Institute, University of Utah, Salt Lake City, UT, USA

${ }^{i}$ Department of Internal Medicine, Hematology-Oncology Division, University of Texas Southwestern Medical Center, Dallas, TX, USA

${ }^{\mathrm{j}}$ Asan Medical Center, Seoul, South Korea

${ }^{\mathrm{k}}$ Aarhus University Hospital, Aarhus, Denmark

${ }^{1}$ University Hospitals Leuven, Leuven, Belgium

${ }^{\mathrm{m}}$ National and Kapodistrian University of Athens, Athens, Greece

${ }^{\mathrm{n}}$ Cleveland Clinic Taussig Cancer Institute, Cleveland, $\mathrm{OH}, \mathrm{USA}$

${ }^{\circ}$ Princess Margaret Cancer Centre, Toronto, ON, Canada

${ }^{\mathrm{p}}$ Yonsei Cancer Center, Yonsei University College of Medicine, Seoul, South Korea

${ }^{\mathrm{q}}$ National Cancer Centre Singapore, Singapore, Singapore

\section{Abstract.}

Background: Fourth-line therapy (4LT) in the treatment of metastatic renal cell carcinoma (mRCC) varies significantly due to the lack of data and recommendations to guide treatment decisions.

Objective: To evaluate the use and efficacy of 4LT in mRCC patients.

Methods: The International mRCC Database Consortium (IMDC) dataset was used to identify patients with mRCC treated with 4LT. This is a multicenter, retrospective cohort study. Overall survival (OS) and progression-free survival (PFS) were calculated using Kaplan-Meier curves. Patients were evaluated for overall response. The six prognostic variables included in

\footnotetext{
${ }^{1}$ This article received a correction notice (Erratum) with the reference: 10.3233/KCA-200005, available at https://content.ios press.com/articles/kidney-cancer/kca200005.

${ }^{2}$ Contributed equally as senior authors.
} 
the IMDC prognostic model were used to stratify patients into favorable-, intermediate- and poor-risk groups. Exploratory analyses were performed examining the elderly (>70 years old) and non-clear cell RCC subgroups. Proportional hazards regression modelling was performed adjusting these covariates by IMDC criteria measured at initiation of 4th line therapy. Results: 7498 patients were treated with first line targeted therapy and out of these 594 (7.9\%) received 4LT. Everolimus was the most frequently used 4LT (16.8\%). Sorafenib, axitinib, pazopanib, sunitinib and clinical trial drugs were also used in $>10 \%$ of patients. The OS of patients on any 4LT was 12.8 months, with a PFS of 4.4 months. The overall response rate (ORR) was $13.7 \%$. Favorable-risk patients using IMDC criteria (5\%) displayed an OS of 23.1 months, intermediate-risk patients $(66 \%)$ had an OS of 13.8 months and poor-risk patients $(29 \%)$ had an OS of $7.8(p<0.0001)$ months. Age $>70$ years and non-clear cell histology did not impact OS. Our study is limited by its retrospective design.

Conclusions: 4LT use appears to have activity in mRCC patients. The IMDC continues to be of prognostic value in the fourth-line setting for OS. This study helps to set a benchmark for response rate and survival for which clinical trials can plan sample size calculations and aim to improve upon.

Keywords: Metastatic renal cell carcinoma, fourth line therapy, targeted therapy, checkpoint inhibitors

\section{INTRODUCTION}

With the introduction of several targeted therapies and checkpoint inhibitors, survival outcomes of mRCC patients continue to improve. As of today, two mammalian target of rapamycin (mTOR) inhibitors and six drugs targeting the vascular endothelial growth factor (VEGFR) pathway are approved for mRCC treatment [1]. Additionally, cabozantinib, an inhibitor of VEGFR receptor (VEGFR), MET and AXL, and nivolumab, a programmed cell death-1 (PD- 1) checkpoint inhibitor have also been approved for treatment in $\mathrm{mRCC}[2,3]$.

With the growing number of available therapeutic agents, the optimal treatment patterns in 4LT need to be examined. The European Society for Medical Oncology (ESMO) and the National Comprehensive Cancer Network (NCCN) provide some guidelines for the most appropriate first-, second- and third-line treatment $[4,5]$. However, no robust evidence exists to optimize treatment selection in the 4LT setting. The RECORD-1 and METEOR trials were the only randomized controlled trials to include patients in the fourth-line setting $[3,6]$. The outcomes of patients receiving everolimus or cabozantinib in fourth-line setting were not well characterized as they represent small patient subgroups in each trial $[3,7]$.

As a result, treatment sequences in 4LT vary significantly amongst different centers and jurisdictions. Additionally, many patients do not receive reimbursement for their respective 4LT drugs. This study aims to examine the effects of 4LT on mRCC patients. It also explores if the IMDC prognostic factors can be successfully used to stratify patients into risk categories.

\section{MATERIAL AND METHODS}

\section{Patient population}

Thirty-five international cancer centers in Canada, USA, Denmark, Greece, South Korea, Australia, New Zealand, Japan, Singapore, Italy and Belgium provided patient data. Patients were obtained by pharmacy, registry or consecutive clinic lists and individual retrospective chart reviews were performed to collect patient data. Standardized database templates were used and the data included patients accrued between 2005 and October 8th 2016.

Patients included in this study had $\mathrm{mRCC}$ and were treated with one or more VEGF or mTOR targeted agents. Previous treatment with IFN $\alpha$ or IL2 was not counted as a first line of therapy, because we wanted to focus on targeted therapies. Institutional review board approval was obtained from each center participating in this study.

\section{Statistical analysis}

Statistical analyses were performed using SAS version 9.4 (Cary, NC, USA). PFS and OS were evaluated using Kaplan-Meier curves. OS was defined as time from the start of 4LT to death or censored at last follow-up. PFS was defined as the time from the start of 4LT until death, progression based on the Response Evaluation Criteria in Solid Tumors guidelines, termination of 4LT or censored at last follow up. Exploratory analyses were performed examining subgroups of patients including the elderly (defined as $>70$ years old) and non-clear cell RCC. Proportional hazards regression modelling was performed adjusting these covariates by IMDC criteria measured at initiation of 4 th line therapy. 
Patients were stratified into prognostic groups using the following six factors included in the IMDC prognostic model: (1) Karnofsky Performance Score (KPS) $<80 \%$, (2) time from diagnosis to initiation of targeted therapy $<1$ year, (3) hypercalcemia, (4) anemia, (5) neutrophilia, and (6) thrombocytosis. In the 4LT analysis all variables except for time from diagnosis to initiation of first line targeted therapy $<1$ year were collected at the start of fourth-line therapy. Patients were stratified into IMDC favorable-risk (0 prognostic factors), IMDC intermediate-risk (1-2 prognostic factors) and IMDC poor-risk (3-6 prognostic factors) and analyzed for OS.

\section{RESULTS}

\section{Patient characteristics and treatments}

A total of 7498 patients were included in this analysis. 3854 (51.4\%) patients who were previously treated with first-line therapy moved on to receive second line therapy. $1813(24.2 \%)$ progressed to third-line therapy and $594(7.9 \%)$ patients received 4LT. The median follow-up for the entire cohort was 52.1 months from the initiation of first line therapy. For 4LT patients, the median followup from the initiation of 4LT was 25.7 months. Their baseline characteristics at initiation of 4LT are shown in Table 1. 196/1701 (11.5\%) mRCC patients from the United States received 4LT, while only $398 / 5797$ patients $(7 \%)$ of patients outside of the USA received 4LT $(p<0.0001) .439 / 557(78.8 \%)$ patients had stopped fourth-line therapy and 370/594 patients $(62 \%)$ died when this analysis was conducted. 37 patients were missing information about the continuation of therapy. At the time of analysis $89 \%$ stopped first-line therapy, $88 \%$ stopped second-line therapy, $88 \%$ stopped third-line and $79 \%$ of patients had stopped 4LT. Everolimus was the most common fourth-line therapy $(16.8 \%)$, followed by sorafenib (14.5\%), axitinib (13.1\%), pazopanib (13.0\%), sunitinib (13.0\%), and clinical trial drugs (11.5\%) (Fig. 1). No treatment showed significant superiority in OS or PFS (Table 2).

\section{Survival outcomes, IMDC risk analysis \& Subgroup analysis}

Median OS from initiation of any 4LT was 12.8 months (95\% CI: 1.4-14.4) (Table 2). Median PFS from initiation of any 4LT was 4.4 months (95\% CI: 4.0-5.1) (Table 2). 55.2\% of patients had a best
Table 1

Patient characteristics at initiation of 4LT and 1LT (N-594)

\begin{tabular}{llc}
\hline 4LT Parameter & N & $\%$ \\
\hline Median Age & 63 (IQR: 56-78) \\
Male & $445 / 594$ & 75 \\
IMDC Favorable Risk at initiation of 4LT & $14 / 288$ & 5 \\
IMDC Intermediate Risk at initiation of 4LT & $189 / 288$ & 66 \\
IMDC Poor Risk at initiation of 4LT & $85 / 288$ & 29 \\
<80 KPS & $190 / 542$ & 35 \\
Diagnosis to Targeted Therapy <1yr & $279 / 580$ & 48 \\
Prior Nephrectomy & $540 / 594$ & 91 \\
Hypercalcemia & $39 / 340$ & 11 \\
Anemia & $308 / 401$ & 77 \\
Neutrophilia & $72 / 411$ & 18 \\
Thrombocytosis & $80 / 415$ & 19 \\
Non-clear cell histology & $54 / 490$ & 11 \\
Sarcomatoid features & $47 / 523$ & 9 \\
Brain Metastasis & $24 / 530$ & 5 \\
1LT Parameter & & \\
IMDC Favorable Risk at initiation of 1LT & $106 / 401$ & 26 \\
IMDC Intermediate Risk at initiation of 1LT & $262 / 401$ & 65 \\
IMDC Poor Risk at initiation of 1LT & $33 / 401$ & 8 \\
\hline
\end{tabular}

IMDC = International Metastatic Renal Cell Carcinoma Database Consortium; KPS = Karnofsky Performance Status. 4LT = Fourthline therapy. 1LT = First-line therapy.

response of stable disease or better. Upon stratifying 4LT patients into risk categories $(n=288), 5 \%$ were favorable-risk, $66 \%$ were intermediate-risk, and $29 \%$ were poor-risk patients. Favorable-risk patients had an OS of 23.1 months (95\% CI: 14.7-NR), intermediate-risk patients had an OS of 13.8 months (95\% CI: 11.4-17.5), and poor-risk patients had an OS of 7.8 months (95\% CI: 4.9-12.2) $(p<0.0001)$ (Fig. 2). In an effort to explore subgroups of patients receiving fourth-line therapies, we examined the use of age and non-clear cell RCC as potential prognostic factors for OS. Patients $>70$ years of age had no difference in their OS when adjusting for the IMDC criteria, the hazard ratio was not statistically significant (HR 0.84, 95\% CI: $0.56-1.25, p=0.38$ ). Patients with non-clear cell RCC had no difference in their OS when compared to those with clear cell histology. When adjusting for fourth-line IMDC criteria, the hazard ratio for death was not statistically significant (HR 1.13, 05\% CI: 0.72-1.77, $p=0.61$ ). Other subgroup analyses were not possible due to small patient numbers.

\section{DISCUSSION}

As of today, the ideal 4LT for mRCC patients remains unknown. Most clinical trials are set in the first- and second-line setting. Thus, the fourth-line setting has not been studied extensively. Furthermore, 


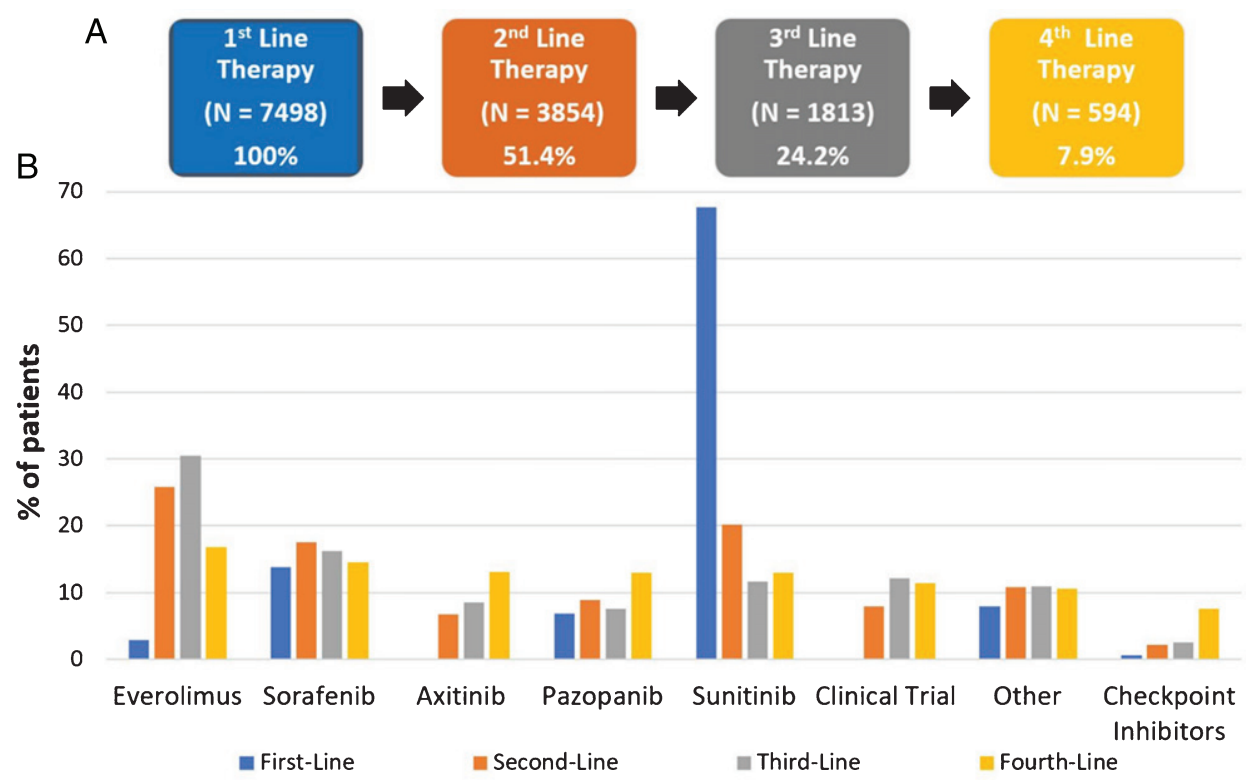

Fig. 1. Total number of patients within the IMDC receiving first-, second-, third- and fourth-line therapy (A). Therapies given in the first-, second-, third- and fourth-line setting to patients who received 4LT $(\mathrm{N}=594)(\mathrm{B})$.

Table 2

Fourth-line progression free survival (PFS)/overall survival (OS)/best response

\begin{tabular}{lcccccc}
\hline Fourth-line Drug & OS (months) & PFS (months) & \multicolumn{4}{c}{ Best Response } \\
\cline { 4 - 7 } & & & PR & SD & PD & CR \\
\hline Everolimus & $3.6(3.0-4.7)$ & $12.8(9.5-18.5)$ & $2 / 68$ & $27 / 68$ & $39 / 68$ & $0 / 68$ \\
Sorafenib & $3.9(2.8-5.3)$ & $11.4(8.9-16.3)$ & $8 / 71$ & $30 / 71$ & $33 / 71$ & $0 / 71$ \\
Axitinib & $7.3(4.0-11.2)$ & $18.0(12.4-22.0)$ & $3 / 46$ & $24 / 46$ & $19 / 46$ & $0 / 46$ \\
Pazopanib & $4.6(3.4-6.8)$ & $9.4(7.3-13.8)$ & $9 / 49$ & $20 / 49$ & $20 / 49$ & $0 / 49$ \\
Sunitinib & $5.3(3.4-6.2)$ & $12.1(8.8-16.1)$ & $16 / 65$ & $24 / 65$ & $25 / 65$ & $0 / 65$ \\
Clinical Trial & $4.4(3.2-5.6)$ & $14.2(9.4-20.6)$ & $4 / 42$ & $20 / 42$ & $18 / 42$ & $0 / 42$ \\
Nivolumab & $13.6(3.9-N R)$ & NR $(14.4-N R)$ & $7 / 23$ & $6 / 23$ & $9 / 23$ & $1 / 23$ \\
Total & $4.4(4.0-5.1)$ & $12.8(1.4-14.4)$ & $49 / 364$ & $151 / 364$ & $163 / 364$ & $1 / 364$ \\
& & & $13.5 \%$ & $41.5 \%$ & $44.8 \%$ & $0.3 \%$ \\
\hline
\end{tabular}

$\mathrm{CR}=$ Complete Response; $\mathrm{PR}=$ Partial Response $\mathrm{SD}=$ Stable Disease $; \mathrm{PD}=$ Progressive Disease .

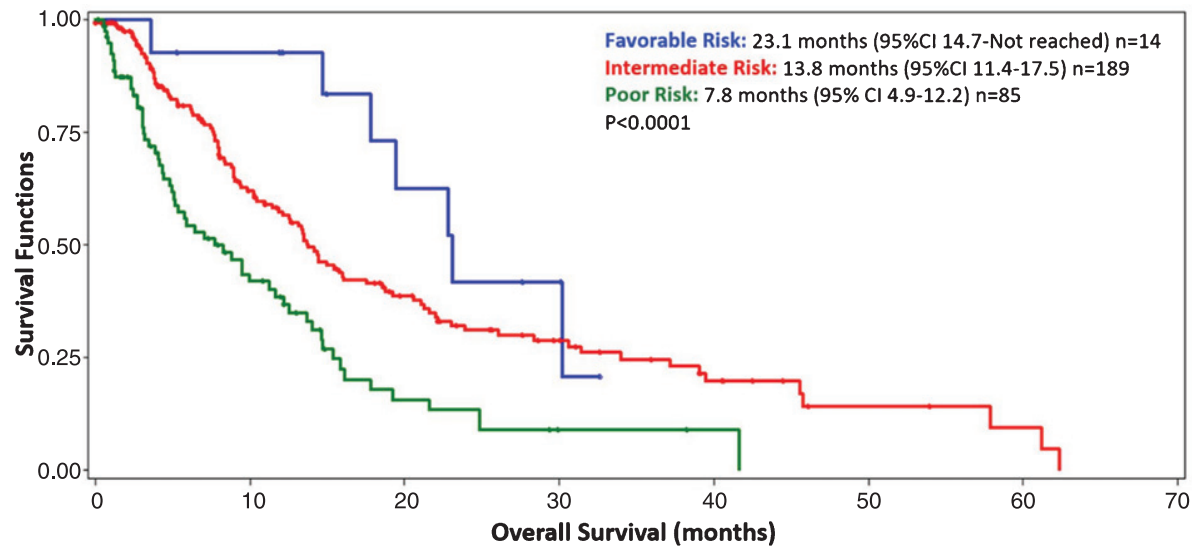

Fig. 2. Kaplan Meier curve depicting the overall survival from the initiation of fourth-line targeted therapy for 288 metastatic renal cell carcinoma patients with complete prognostic information. Blue $=$ favorable risk $(5 \%)$, Red $=$ intermediate risk $(66 \%)$, Green $=$ poor risk $(29 \%)$. Patients were stratified by IMDC prognostic categories: 0 factors $=$ favorable risk, $1-2$ factors $=$ intermediate risk, $3-6$ factors $=$ poor risk. $\mathrm{CI}=$ confidence interval. 
only a handful of retrospective studies have looked at small cohorts of patients receiving 4LT, with many of these including cytokine immunotherapy as a previous line of treatment [8-11]. As a result, no clear guidelines exist to guide clinicians in the fourth-line setting.

Our results confirm a largely heterogeneous patient population receiving different targeted agents. Everolimus was used in $16.8 \%$ of all 4LT patients, while four other treatments were used in 13-14.5\% of mRCC patients. Since currently no robust evidence exists to suggest superiority of any treatment used in the fourth-line setting, clinicians will continue to administer treatments based on institutional preference and availability. For example, Americans with health-insurance may be eligible for more reimbursed approved treatments than non-American patients with universal health coverage who may seek clinical trial involvement instead [12,13]. This may be true because in our study $11.5 \%$ of Americans received $4 \mathrm{LT}$ relative to $7 \%$ of non-Americans.

Our results indicate that $7.9 \%$ of mRCC patients receive 4LT. Median OS from initiation of any 4LT was 12.8 months, while the median PFS from initiation of any 4LT was 4.4 months. The 4LT survival data is comparable to a small $(\mathrm{N}=56)$ fourth-line retrospective study, which displayed an OS and PFS of 10.5 and 3.2 months, respectively [8]. Moreover, the ORR of $13.7 \%$ is comparable to that of two small German retrospective studies, which displayed ORRs of $8.9 \%$ and $16.7 \%[8,10]$.

Our data provides further evidence that patients in fourth-line setting can still experience a substantial survival benefit. With the introduction of novel drugs and reduced treatment toxicities, treatment selection for mRCC patients continues to improve. Identifying the patients that will most likely benefit from 4LT remains crucial to ensure the best possible treatment outcomes. The discovery and use of novel biomarkers in treatment selection will hopefully allow us to elucidate optimal treatment patterns for targeted therapy in first-, second-, third- and fourth-line settings.

The IMDC prognostic criteria appear to be able to stratify patients into favorable-, intermediate- and poor-risk groups in the fourth-line setting for overall survival. The IMDC model has previously been validated in first- and second-line settings, while also stratifying patients appropriately into prognostic groups in the third-line setting [13-15]. Interestingly, our data shows that even some poor risk patients (8\%) at the initiation of first-line therapy received 4LT. As a result, the IMDC criteria cannot help to identify patients at the outset of receiving first-line therapy which patient will move on to 4LT.

Our study is limited by its retrospective design. A selection bias exists for 4LT patients, since patients with high disease burden and rapidly progressing disease may not receive multiple lines of targeted agents. We tried to address this bias by using consecutive patient data from hospital records. We stratified patients into prognostic groups by using the IMDC prognostic factors. However, some prognostic factors were missing. Further studies need to be conducted to explore the effect of 4LT.

Finally, with the increasing use of checkpoint inhibitors and newer drugs that target mechanisms of VEGF resistance (e.g. cabozantinib and lenvantinib), the fourth-line treatment landscape will continue to evolve. Novel studies, such as NCT02071862 and NCT02724020 testing the Glutaminase Inhibitor CB-839 and MLN0128/MLN1117, respectively, will allow for the enrollment of patients in fourth-line setting $[16,17]$. With more clinical trials beginning to include fourth-line patients, it will be interesting to see if outcomes improve or if the efficacy of 4LT is dependent upon which types of prior therapies patients receive. This study helps to set a benchmark for response rate and survival for which clinical trials can plan sample size calculations and aim to improve upon.

\section{CONCLUSIONS}

With an increasing number of therapeutic agents and improving treatment algorithms the guidelines for mRCC treatment will continue to evolve. Our study displayed that 4LT drug selection was highly heterogeneous. The IMDC prognostic factors can stratify patients into favorable-, intermediate- and poor-risk groups in the fourth-line setting. 4LT appears to benefit a select group of patients. Further studies need to be conducted to identify groups that will most likely benefit from 4LT.

\section{CONFLICTS OF INTEREST}

Choueiri: Research funding (AstraZeneca, BMS, Exelixis, Genentech, GSK, Merck, Novartis, Peloton, Pfizer, Roche, Tracon, Eisai), Consulting or Advisory Role (AstraZeneca, Bayer, BMS, Cerulean, Eisai, Foundation Medicine Inc., ExelixisGenentech/Roche, GlaxoSmithKline, Merck, Novartis, Peloton, Pfizer, Prometheus Labs). 
Donskov: Research funding (Pfizer, Novartis)

Lalani: Educational Grant (Pfizer)

Bamias: Honoraria, Advisory Role and Research Funding (Roche, Pfizer, Novartis, BMS)

Porta: Consulting and/or Speaker (Novartis, BMS, Pfizer, Eisai, Ipsen, Janssen, EUSA, Peloton)

Beuselinck: Honoraria (amgen, Pfizer, Janssen, Bayer), investigator of EudraCT: 2011-00608540/MetaSun trial supported by Pfizer

Agarwal: Consulting (Pfizer, Exelixis, Argos, Cerulean)

Rini: Consulting and Research funding (Pfizer)

Kanesvaran: Honoraria (Novartis, Pfizer, Bayer)

Heng: Consulting (Pfizer, Novartis, Bristol-Myers Squibb, Janssen Pharmaceuticals, Astellas Pharma)

Lee: Honoraria (Pfizer and Astellas), consulting (Astellas), Research funding (Pfizer, Bayer, Janssen, Novartis, and Exelixis)

Stukalin, Wells, Srinivas, Sim, Bowman, Brugarolas, Pasini, Fraccon, Rha: Nothing to disclose

\section{REFERENCES}

[1] Stukalin I, Alimohamed N, Heng DYC. Contemporary Treatment of Metastatic Renal Cell Carcinoma. Oncol Rev 2016;10:295.

[2] Motzer RJ, Rini BI, McDermott DF, et al. Nivolumab for Metastatic Renal Cell Carcinoma: Results of a Randomized Phase II Trial. J Clin Oncol 2015;33:1430-7.

[3] Choueiri TK, Escudier B, Powles T, et al. Cabozantinib versus Everolimus in Advanced Renal-Cell Carcinoma. N Engl J Med 2015;373:1814-23.

[4] Escudier B, Porta C, Schmidinger M, et al. Renal cell carcinoma: ESMO Clinical Practice Guidelines for diagnosis, treatment and follow-up. DOI: 10.1093/annonc/mdw328

[5] NCCN Clinical Practice Guidelines in Oncology (NCCN Guidelines (B) Kidney Cancer NCCN.org https://www.nccn. org/professionals/physician_gls/pdf/kidney.pdf (2017, accessed 21 April 2017).

[6] Motzer RJ, Escudier B, Oudard S, et al. Efficacy of everolimus in advanced renal cell carcinoma: A doubleblind, randomised, placebo-controlled phase III trial. Lancet (London, England) 2008;372:449-56.
[7] Motzer RJ, Escudier B, Oudard S, et al. Phase 3 trial of everolimus for metastatic renal cell carcinoma. Cancer 2010;116:4256-65.

[8] Ralla B, Erber B, Goranova I, et al. Efficacy of fourthline targeted therapy in patients with metastatic renal cell carcinoma: A retrospective analysis. World J Urol 2016;34:1147-54.

[9] Maute L, Grünwald V, Weikert S, et al. Therapy of mRCC beyond mTOR-inhibition in clinical practice: Results of a retrospective analysis. J Cancer Res Clin Oncol 2014;140:823-7.

[10] Vallet S, Pahernik S, Höfner T, et al. Efficacy of Targeted Treatment Beyond Third-Line Therapy in Metastatic Kidney Cancer: Retrospective Analysis From a Large-Volume Cancer Center. Clin Genitourin Cancer 2015;13:e145-e152.

[11] Soerensen AV, Donskov F, Hermann GG, et al. Improved overall survival after implementation of targeted therapy for patients with metastatic renal cell carcinoma: Results from the Danish Renal Cancer Group (DARENCA) study-2. Eur J Cancer 2014;50:553-62.

[12] Jones R, DeSantis M. Access to Targeted Therapies in Renal Cell Cancer. Semin Oncol 2013;40:521-528.

[13] Wells JC, Stukalin I, Norton C, et al. Third-line Targeted Therapy in Metastatic Renal Cell Carcinoma: Results from the International Metastatic Renal Cell Carcinoma Database Consortium. Eur Urol. Epub ahead of print June 2016. DOI: 10.1016/j.eururo.2016.05.049

[14] Heng DYC, Xie W, Regan MM, et al. Prognostic Factors for Overall Survival in Patients With Metastatic Renal Cell Carcinoma Treated With Vascular Endothelial Growth Factor-Targeted Agents: Results From a Large, Multicenter Study. J Clin Oncol 2009;27:5794-99.

[15] Ko JJ, Xie W, Kroeger N, et al. The International Metastatic Renal Cell Carcinoma Database Consortium model as a prognostic tool in patients with metastatic renal cell carcinoma previously treated with first-line targeted therapy: A population-based study. Lancet Oncol 2015;16:293-300.

[16] Study of the Glutaminase Inhibitor CB-839 in Solid Tumors - Full Text View - ClinicalTrials.gov https:// clinicaltrials.gov/ct2/show/NCT02071862

[17] MLN0128 and MLN0128+MLN1117 Compared With Everolimus in the Treatment of Adults With Advanced or Metastatic Clear-Cell Renal Cell Carcinoma - ClinicalTrials.gov https://clinicaltrials.gov/ct2/show/NCT02724020 